\title{
Interferon-alfa in the treatment of patients with inoperable locally advanced or metastatic renal cell carcinoma: a systematic review
}

\author{
Christina Canil, MD, FRCPC; ${ }^{*}$ Sebastien Hotte, MD, FRCPC; ${ }^{\dagger}$ Linda A. Mayhew, PhD; ${ }_{+}^{\ddagger}$ Tricia S. Waldron; \\ Eric Winquist, MD, FRCPC ${ }^{\S}$
}

\begin{abstract}
A systematic review was undertaken to determine whether interferon-alfa (IFN- $\alpha$ ) is an effective treatment for patients with inoperable locally advanced or metastatic renal cell carcinoma (mRCC). MEDLINE, EMBASE, the Cochrane Library, guideline databases and relevant meeting proceedings were searched. Randomized clinical trials (RCTs) or meta-analyses comparing IFN- $\alpha$-containing regimens to placebo or non-immunotherapy controls, and that reported response rate, survival, toxicity or quality of life data were eligible. Two systematic reviews and eight RCTs met the selection criteria. A Cochrane review updated in 2005 reported higher response rates and reduced one-year mortality based on 4 RCTs in patients who received IFN- $\alpha$. Of the eight RCTs, three reporting objective response rate showed significant differences favouring IFN- $\alpha$. Two of five trials reporting survival data showed longer median survival in the IFN- $\alpha$ group. Adverse effects of IFN- $\alpha$ were consistent across the trials with increased intensity and frequency concordant with increased IFN- $\alpha$ dose. Meta-analyses of seven RCTs for objective response and six RCTs for mortality favoured IFN- $\alpha$ : odds ratio 6.87 (95\% Confidence Interval $[\mathrm{Cl}], 3.29$ to 14.35$)$ and hazard ratio $0.79(95 \% \mathrm{Cl}, 0.69$ to 0.91$)$, respectively. The effectiveness of IFN- $\alpha$ in mRCC has been subject to skepticism. As IFN- $\alpha$ has been used as a control arm in RCTs of new targeted therapies, therapies which not all patients may have access to, information about its effectiveness remains relevant. These data confirm genuine, if modest, effectiveness of IFN- $\alpha$ in mRCC.
\end{abstract}

\section{Résumé}

Une revue systématique a été entreprise afin de déterminer si l'interféron alpha (IFN- $\alpha$ ) représente un traitement efficace chez les patients atteints d'un hypernéphrome localement avancé ou métastatique et non opérable. Des recherches ont été effectuées dans les bases de données MEDLINE et EMBASE, dans la Bibliothèque Cochrane, les guides de pratique et les comptes rendus des réunions pertinentes. Les essais cliniques avec randomisation (ECR) ou les méta-analyses comparant des schémas contenant I'IFN- $\alpha$ à un placebo ou à un traitement témoin autre qu'une immunothérapie et qui faisaient état du taux de réponse, du taux de survie, des données sur la toxicité ou la qualité de vie pouvaient être inclus. Deux examens systématiques et huit ECR satisfaisaient aux critères de sélection. Une revue provenant de la Bibliothèque Cochrane et mise à jour en 2005 signalait des taux de réponses plus élevés et une mortalité réduite après un an en fonction de 4 ECR chez des patients ayant reçu de I'IFN- $\alpha$. Sur les huit ECR retenus, trois faisant état du taux de réponse objective ont montré une différence significative en faveur de I'IFN- $\alpha$. Sur les cinq essais faisant état de données sur la survie, deux ont montré une survie médiane plus longue dans le groupe sous IFN- $\alpha$. Les effets indésirables de I'IFN- $\alpha$ étaient constants d'un essai à l'autre, l'intensité et la fréquence de ces effets variant en fonction de la dose d'IFN- $\alpha$. Les métaanalyses de sept ECR pour dégager la réponse objective et de six ECR pour dégager les taux de mortalité étaient en faveur de l'IFN$\alpha$ : rapport de cotes, 6,87 (intervalle de confiance [IC] à $95 \%$, 3,29 à 14,35) et rapport des risques instantanés, 0,79 (IC à $95 \%$, $0,69$ à 0,91$)$, respectivement. L'efficacité de I'IFN- $\alpha$ dans le traitement de l'hypernéphrome métastatique est parfois mise en doute. Comme I'IFN- $\alpha$ a été utilisé comme traitement témoin dans des ECR portant sur de nouveaux traitements ciblés, auxquels tous les patients n'ont pas nécessairement accès, les renseignements concernant son efficacité conservent toute leur pertinence. Ces données confirment l'efficacité réelle, quoique modeste, de I'IFN- $\alpha$ dans le traitement de I’hypernéphrome métastatique.

Can Urol Assoc J 2010;4(3):201-8

\section{Introduction}

In 2009, about 4600 new renal cell carcinoma (RCC) cases and 1600 RCC deaths were projected for Canada. ${ }^{1}$ Among patients with RCC at the time of their first diagnosis, $45 \%$ would present with localized disease, 25\% would have locally advanced disease with lymph node or local organ involvement and the remaining $30 \%$ would present with metastases. ${ }^{2}$

Patients who present with localized disease are best treated with surgery, but as many as $30 \%$ of these patients will eventually relapse. ${ }^{3}$ When patients present with or develop inoperable locally advanced or metastatic disease, the main intent of treatment is to effectively control symptoms and provide a chance of improved survival. Unfortunately, the treatment of late-stage RCC remains a challenge to oncologists and urologists; unlike other solid malignancies, advanced or metastatic RCC is highly resistant to most available chemotherapeutic agents. ${ }^{4,5}$

Immunotherapy was first suggested as a treatment for advanced or metastatic RCC after occasional spontaneous tumour regressions, and the presence of anti-tumour immune responses were observed in patients with this neoplasm. ${ }^{6}$ 
The major immunological approaches that have been investigated in these patients have included cytokines, either as single agents or in combination with other cytokines or chemotherapy. ${ }^{7}$ One of the first classes of cytokines to be evaluated was interferon. Interferons are naturally occurring glycoproteins that are produced in response to viral infections, antigens, and mitogens and are often induced by other cytokines like tumour necrosis factor (TNF) and interleukins. ${ }^{8}$ The anti-tumour activity of interferons is mediated by various mechanisms, such as immunomodulation, antiproliferative activity, inhibition of angiogenesis, regulation of differentiation, interaction with growth factors and modulation of gene expression. ${ }^{8}$ This systematic review assesses the effectiveness of interferon-alfa (IFN- $\alpha$ ) for the treatment of advanced or metastatic RCC, based on the results of reported randomized controlled trials (RCTs).

\section{Methods}

\section{Literature search strategy}

Relevant RCTs, controlled clinical trials, meta-analyses, systematic reviews and practice guidelines were identified in electronic searches of MEDLINE (1966 through May 2009) and EMBASE (1980 through 2009 week 19).

Searches were also made in the Cochrane Library (2009, Issue 2), meeting proceedings of the American Society of Clinical Oncology (ASCO) 1995-2008, the ASCO genitourinary symposia (2008-2009), the American Urological Association (1995-2009); the Canadian Medical Association Infobase, and the National Guidelines Clearing House for relevant articles, abstracts, and practice guidelines.

\section{Study selection criteria}

Relevant articles and abstracts were selected by 4 reviewers. Papers were included if they were published RCTs, abstracts of RCTS, or meta-analyses that compared IFN- $\alpha$ containing treatment regimens to regimens without IFN- $\alpha$ in patients with inoperable locally advanced or metastatic RCC. Comparison groups could include placebo, cytotoxic chemotherapy, hormonal therapies, such as medroxyprogesterone (MPA), and IFN- $\gamma$. Reports were required to provide data on at least one of the following outcomes: response rate, survival (overall, progression-free, and time-to-progression), toxicity and quality of life.

Studies that compared surgery or radiotherapy with IFN- $\alpha$-containing treatment, compared IFN- $\alpha$ with angiogenesis inhibitors, or compared IFN- $\alpha$ with interleukin-2 (IL-2) were excluded, as these are examined in separate systematic reviews.

\section{Synthesizing the evidence}

For some eligible trials, odds ratios (OR) for overall mortality at 1 year and objective response and hazard ratios $(\mathrm{HR})$ for overall mortality were available from a Cochrane meta-analysis by Coppin and colleagues. ${ }^{9}$ The analytic plan was to combine published data on these endpoints for all eligible trials, using meta-analysis. When the HR and its associated variance were available, those statistics were either extracted directly from the trial itself, from the Cochrane meta-analysis ${ }^{9}$ or were obtained through personal communication with trial authors. Otherwise, the HR was estimated indirectly from data extracted from published KaplanMeier curves, ${ }^{10-12}$ using the methods of Parmar and colleagues. ${ }^{13}$ If data were not provided from which the HR could be derived or the authors did not provide the HR, the trial was not included in the meta-analysis. To estimate the overall effect of IFN- $\alpha$, the data were combined using Review Manager version 4.2 (Cochrane Collaboration 2002, Review Manager, Oxford, England). Results are expressed as $\mathrm{HR}$ or OR with $95 \%$ confidence intervals $(\mathrm{Cl})$, where values $<1.0$ represent a benefit for IFN- $\alpha$ over the alternative (for HR and OR of mortality), and values $>1.0$ indicate a benefit for IFN- $\alpha$ (for OR of response). Use of a random effects model was planned.

\section{Results}

\section{Literature search results}

Two systematic reviews with meta-analyses ${ }^{9,14}$ and 8 RCTs met the inclusion criteria. ${ }^{10-12,15-22}$ No evidence-based guidelines were identified.

\section{Systematic reviews with meta-analyses}

The evidence from a meta-analysis published in $1999^{14}$ was superseded by the results of a Cochrane review by Coppin and colleagues. ${ }^{9}$

Within the Cochrane review, the pooled results of 4 RCTs showed that IFN- $\alpha$ was associated with reduced 1-year mortality and greater remission rates compared with control therapy (MPA or vinblastine [VBL]). Remission, defined as the number of patients receiving a partial or complete response, was greater for IFN- $\alpha$ than control $(12.5 \%$ vs. $1.5 \%)$ with a pooled OR of $7.61(95 \% \mathrm{Cl}, 3.02-19.2)$. Interferon- $\alpha$ was also associated with reduced 1 -year mortality $(\mathrm{OR}=0.56$; $95 \% \mathrm{Cl}, 0.40-0.77)$.

The 4 trials were also pooled using the methods of Parmar and colleagues to further explore the effect of IFN- $\alpha$ on mortality outcomes. ${ }^{13}$ The pooled overall HR for death was 0.74 $(95 \% \mathrm{Cl}, 0.63-0.88)$, indicating a survival benefit for IFN- $\alpha$ 
over control therapy. The authors concluded that IFN- $\alpha$ demonstrated a modest improvement in remission rates and a consistent and statistically significant mortality reduction compared with a variety of controls. The analysis that compared trials using the recombinant subtypes IFN- $\alpha-2 a$ and IFN- $\alpha-2 b$ showed no evidence of statistical heterogeneity for either objective response or 1-year mortality.

\section{Randomized controlled trials}

Eight RCTs comparing IFN- $\alpha$ either alone or plus control therapy with control therapy alone met the inclusion criteria. ${ }^{10-12,15-22}$ The trials included 1360 patients, with patient accrual per trial arm ranging from 16 to 176 . Patients were eligible for inclusion if they had histologically confirmed RCC and showed no signs of brain metastases. The median age of patients ranged from 56 to 63 years, and most were men (range, $59 \%$ to $75 \%$ ) with good performance status (i.e., Eastern
Cooperative Oncology Group or World Health Organization $<2$, Karnofsky $>80 \%$ or Zubrod $<2$ ). The 8 RCTs provided a total of 17 comparisons. The RCTs reported outcome data for objective response rate, survival, disease progression and toxicity. No RCTs formally assessed quality of life.

\section{Trial quality}

Based on their reports, the quality of the trials was generally suboptimal. There were inconsistencies in data reporting in several of the trials. Two studies did not report the number of patients randomized per group. ${ }^{15,16}$ In addition, there were inconsistencies within a report regarding the number of patients reported, treated and randomized; $10-12,16,18,19$ in some cases, different publications of the same trial reported a different number of patients randomized. Some of these issues were resolved through personal correspondence either with the study authors or with the author of the Cochrane

\begin{tabular}{|c|c|c|c|c|c|c|c|c|c|c|}
\hline Trial & $\begin{array}{l}\text { Description } \\
\text { of random } \\
\text { allocation }\end{array}$ & Design & $\begin{array}{l}\text { Non-inferiority } \\
\text { margin }\end{array}$ & Power & $\begin{array}{l}\text { Planned } \\
\text { sample } \\
\text { size }\end{array}$ & $\begin{array}{l}\text { Sample } \\
\text { size met? }\end{array}$ & $\begin{array}{l}\text { Intention- } \\
\text { to-treat } \\
\text { analysis }\end{array}$ & $\begin{array}{c}\text { Per } \\
\text { protocol } \\
\text { analysis? }\end{array}$ & $\begin{array}{c}\text { Details of } \\
\text { withdrawals } \\
\text { and exclusions }\end{array}$ & Blinding \\
\hline $\begin{array}{l}\text { Negrier } \\
2005 / 2006 / 2007^{10-12}\end{array}$ & No & $\begin{array}{c}2 \times 2 \\
\text { factorial }\end{array}$ & $10 \%$ & $\begin{array}{c}80 \%, \\
5 \% \alpha \\
\text { error } \\
\beta=20 \%\end{array}$ & 456 & Yes & Yes & Yes & No & NA \\
\hline $\begin{array}{l}\text { Dutcher } \\
2003^{16}\end{array}$ & No & $\begin{array}{l}\text { Randomized } \\
\text { phase II } \\
\text { 2-stage } \\
\text { design for } \\
\text { each arm }\end{array}$ & NA & NR & NR & NR & NR & NR & No & NA \\
\hline $\begin{array}{l}\text { Hancock } \\
2003^{18,19}\end{array}$ & Yes & $\begin{array}{l}\text { Multicentre } \\
\text { randomized } \\
\text { trial, group- } \\
\text { sequential } \\
\text { analysis } \\
\text { with } \\
\text { triangular } \\
\text { design }\end{array}$ & $12 \%$ & $\begin{array}{c}90 \% \\
5 \% \\
\text { signifi- } \\
\text { cance }\end{array}$ & $\begin{array}{l}\text { Maxi- } \\
\text { mum } \\
\text { sample } \\
\text { size } 600\end{array}$ & $\begin{array}{l}350 \\
\text { recruited* }\end{array}$ & Yes & NR & No & NA \\
\hline $\begin{array}{l}\text { Pyrhönen } \\
1999^{21}\end{array}$ & No & $\begin{array}{l}\text { Phase III } \\
2 \text { arms }\end{array}$ & NA & $\begin{array}{c}80 \% \\
\text { One- } \\
\text { sided } \\
\text { Two- } \\
\text { sided } \\
\text { analyses } \\
\text { used }\end{array}$ & 160 & Yes & Yes & No & NA & NA \\
\hline $\begin{array}{l}\text { Kriegmair } \\
1995^{20}\end{array}$ & No & 2 arms & NA & NR & NR & NR & NR & NR & $\begin{array}{l}\text { Exclusions yes, } \\
\text { withdrawals no }\end{array}$ & NA \\
\hline $\begin{array}{l}\text { Steineck } \\
1990^{22}\end{array}$ & No & 2 arms & NA & NR & NR & NR & No & Unclear & NR & NR \\
\hline $\begin{array}{l}\text { Dexeus } \\
1989^{15}\end{array}$ & No & 2 arms & $30 \%$ & $\begin{array}{c}\text { Power } \\
0.7, \\
\text { signifi- } \\
\text { cance } 0.1\end{array}$ & 32 & Yes & NR & NR & NR & NA \\
\hline $\begin{array}{l}\text { Foon } \\
1988^{17}\end{array}$ & Yes & 3 arms & NA & NR & NR & NR & NR & NR & NR & NR \\
\hline
\end{tabular}

*Trial stopped early on advice of independent data monitoring committee. NA = not applicable; NR = not reported. 
Canil et al.

Table 2. Trial descriptions and outcomes: IFN- $\alpha$ containing regimens vs. control (8 RCTs, 8 comparisons)

\begin{tabular}{|c|c|c|c|c|c|c|c|c|c|c|c|c|c|}
\hline \multirow[t]{2}{*}{ Trial } & \multirow[t]{2}{*}{$\begin{array}{l}\text { Treatment } \\
\text { arms }\end{array}$} & \multirow[t]{2}{*}{$\begin{array}{l}\text { Route, dose and } \\
\text { schedule }\end{array}$} & \multirow{2}{*}{$\begin{array}{c}\text { No. patients } \\
\text { randomized } \\
\text { (evaluable) }\end{array}$} & \multicolumn{4}{|c|}{ Objective response rate \% } & \multicolumn{4}{|c|}{ Survival } & \multicolumn{2}{|c|}{$\begin{array}{l}\text { Progression-free } \\
\text { survival }\end{array}$} \\
\hline & & & & OR & CR & PR & $\begin{array}{c}p- \\
\text { value }^{a}\end{array}$ & $\begin{array}{l}\text { Median } \\
\text { (mos) }\end{array}$ & $\begin{array}{c}p- \\
\text { value }^{a}\end{array}$ & $1-y r, \%$ & $5-y r, \%$ & $\begin{array}{l}\text { Median } \\
\text { (mos) }\end{array}$ & $\begin{array}{c}p- \\
\text { value }^{a}\end{array}$ \\
\hline \multirow[t]{2}{*}{$\begin{array}{l}\text { Negrier } \\
2005 / 2006^{b} / \\
2007^{10-12}\end{array}$} & IFN & sc $9 \mathrm{MU}$ tiw & $122(115)$ & $\begin{array}{l}4.4^{\mathrm{c}} \\
8.7^{\mathrm{d}}\end{array}$ & $\begin{array}{l}0.9^{\mathrm{c}} \\
0.4^{\mathrm{d}}\end{array}$ & $\begin{array}{l}3.5^{c} \\
2.5^{d}\end{array}$ & \multirow[b]{2}{*}{ NR } & $\begin{array}{c}15.2 \\
\text { (range } \\
12.8- \\
19.9 \text { ) }\end{array}$ & \multirow[b]{2}{*}{ NS } & $62.7^{\mathrm{e}}$ & \multirow[b]{2}{*}{ NR } & 3.4 & \multirow[b]{2}{*}{ NR } \\
\hline & MPA & po $200 \mathrm{mg} / \mathrm{d}$ & $123(120)$ & $\begin{array}{l}2.5^{\mathrm{c}} \\
1.6^{\mathrm{d}}\end{array}$ & $\begin{array}{l}0.8^{\mathrm{c}} \\
1.3^{\mathrm{d}}\end{array}$ & $\begin{array}{l}1.7^{\mathrm{c}} \\
6.6^{\mathrm{d}}\end{array}$ & & $\begin{array}{c}14.9 \\
\text { (range } \\
11.7- \\
19.2 \text { ) }\end{array}$ & & $59.5^{\mathrm{e}}$ & & 3.0 & \\
\hline \multirow[t]{2}{*}{ Dutcher, $2003^{16}$} & $\begin{array}{l}\text { IFN-Y + } \\
\text { IFN- } \alpha\end{array}$ & $\begin{array}{l}\text { iv } 0.3 \mathrm{mg} / \mathrm{m}^{2} \\
\text { daily } \times 5 \mathrm{~d} \\
\text { repeated q } 3 \text { wks } \\
\text { sc } 10 \mathrm{MU} / \mathrm{m}^{2} \\
\text { daily }\end{array}$ & NR (49) & 10 & 4 & 6 & \multirow[t]{2}{*}{ NR } & 10.9 & \multirow[t]{2}{*}{ NR } & $47^{e}$ & NR & $\begin{array}{c}\text { FFS } \\
2.9\end{array}$ & \multirow[t]{2}{*}{ NR } \\
\hline & IFN-Y & $\begin{array}{l}\mathrm{sc} 0.1 \\
\mathrm{mg} / \mathrm{m}^{2} / \mathrm{wk} \\
\times 6 \mathrm{wks}\end{array}$ & NR (39) & 0 & 0 & 0 & & 7.0 & & $39^{e}$ & NR & $\begin{array}{c}\text { FFS } \\
1.4\end{array}$ & \\
\hline \multirow[t]{2}{*}{ Hancock, $2003^{18,19}$} & IFN- $\alpha$ & $\begin{array}{l}\text { sc } 10 \mathrm{MU} \text { tiw } \\
\times 12 \text { wks }\end{array}$ & 174 (167) & $14^{f}$ & NR & NR & \multirow{2}{*}{0.001} & 9.0 & \multirow{2}{*}{0.013} & 43 & NR & 4 & \multirow{2}{*}{ NR } \\
\hline & MPA & $\begin{array}{l}\text { po } 300 \mathrm{mg} \mathrm{q} \mathrm{d} \\
\text { x } 12 \text { wks }\end{array}$ & $176(168)$ & $1^{f}$ & NR & NR & & 6.8 & & 32 & NR & 3 & \\
\hline \multirow[t]{2}{*}{ Pyrhönen, 1999²1 } & $\begin{array}{l}\text { IFN- } \alpha 2 a+ \\
\text { VBL }\end{array}$ & $\begin{array}{l}\text { sc or im } 3 \mathrm{MU} \\
\text { tiw for } 1 \mathrm{wk} \\
\text { then sc } 18 \mathrm{MU} \\
\text { tiw }^{\mathrm{g}} \\
\text { iv } 0.1 \mathrm{mg} / \mathrm{kg} \mathrm{q} \\
3 \mathrm{wks}\end{array}$ & 79 (79) & 16.5 & 8.9 & 7.6 & \multirow[t]{2}{*}{0.0025} & 15.5 & \multirow[t]{2}{*}{0.0049} & 55.7 & 4.1 & 3 & \multirow[t]{2}{*}{0.0001} \\
\hline & VBL & $\begin{array}{l}\text { iv } 0.1 \mathrm{mg} / \mathrm{kg} \mathrm{q} \\
3 \mathrm{wks}\end{array}$ & $81(81)$ & 2.5 & 1.2 & 1.2 & & 8.7 & & 38.3 & 0 & 2 & \\
\hline \multirow[t]{2}{*}{ Kriegmair, $1995^{20}$} & $\begin{array}{l}\text { IFN- } \alpha+ \\
\text { VBL }\end{array}$ & $\begin{array}{l}\text { sc } 8 \mathrm{MU} \mathrm{tiw}^{\mathrm{h}} \\
\text { iv } 0.1 \mathrm{mg} / \mathrm{kg} \mathrm{q} \\
3 \mathrm{wks}\end{array}$ & $44(41)$ & 20 & 9 & 11 & \multirow[t]{2}{*}{0.001} & \multirow[t]{2}{*}{ NR } & \multirow[t]{2}{*}{ NS } & $60^{\mathrm{e}}$ & NR & NR & \multirow[t]{2}{*}{ NR } \\
\hline & MPA & $\mathrm{im} 500 \mathrm{mg} / \mathrm{wk}$ & $45(35)$ & 0 & 0 & 0 & & & & $30^{e}$ & NR & NR & \\
\hline \multirow[t]{2}{*}{ Steineck, $1990^{22}$} & IFN- $\alpha 2 a$ & $\begin{array}{l}\text { im } 10 \mathrm{MU} / \mathrm{m}^{2} \\
\text { tiwi dose was } \\
\text { escalated wkly } \\
\text { by } 2.5 \mathrm{MU} / \mathrm{m}^{2} \\
\text { to a } \mathrm{max} \text { of } \\
20 \mathrm{MU} / \mathrm{m}^{2}\end{array}$ & 30 (NR) & 6 & 3 & 3 & \multirow[t]{2}{*}{ NR } & \multirow[t]{2}{*}{ NR } & NR & $30^{e}$ & NR & NR & NR \\
\hline & MPA & $\begin{array}{l}\text { im } 1 \mathrm{~g} \text { tiw } \times 5 \\
\text { wks; } 1 \mathrm{~g} / \mathrm{wk}\end{array}$ & 30 (NR) & 3 & 3 & 0 & & & & $26^{e}$ & NR & NR & \\
\hline Dexeus, $1989^{15}$ & IFN- $\alpha$ & $\begin{array}{l}\operatorname{im} 3 \mathrm{MU} / \mathrm{m}^{2} \\
\mathrm{~d} 1,15 ; 5 \\
\mathrm{MU} / \mathrm{m}^{2} \mathrm{~d} 2,16 \\
10 \mathrm{MU} / \mathrm{m}^{2} \mathrm{~d}, \\
3-5,17-19^{\mathrm{j}}\end{array}$ & NR (16) & 12 & 0 & 12 & & 8.8 & & & & & \\
\hline & $\begin{array}{l}5-F U_{+} \\
\mathrm{CIS}+ \\
\text { DOX+ } \\
\text { MMC } \\
\text { (FAMP) }\end{array}$ & $\begin{array}{l}\text { iv } 750 \mathrm{mg} / \mathrm{m}^{2} \\
\text { d } 1-5 \\
\text { iv } 75 \mathrm{mg} / \mathrm{m}^{2} \\
\text { d } 1^{\mathrm{k}} \\
\text { iv } 25 \mathrm{mg} / \mathrm{m}^{2} \\
\text { d } 2,3 \\
\text { iv } 5 \mathrm{mg} / \mathrm{m}^{2} \\
\text { d } 4,5, \\
\text { Cycle repeated } \\
\text { every } 28 \mathrm{~d}\end{array}$ & NR (16) & 12 & 6 & 6 & NR & 9.1 & NR & NR & NR & NR & NR \\
\hline Foon $1988^{17}$ & IFN- $\alpha$ & $\mathrm{sc} 2 \mathrm{MU} / \mathrm{m}^{2}$ tiw & $21(21)$ & & & & & & & & & & \\
\hline & IFN-Y & sc $1 \mathrm{MU} / \mathrm{m}^{2}$ tiw & $21(21)$ & & $2 \mathrm{PR}$ & & NR & NR & NR & NR & NR & NR & NR \\
\hline & $\begin{array}{l}\text { IFN- } \alpha+ \\
\text { IFN- } \gamma^{\prime}\end{array}$ & & $47(47)$ & & $2 \mathrm{CR}^{\mathrm{m}}$ & & & & & & & & \\
\hline $\begin{array}{l}5-\mathrm{FU}=5 \text {-fluorouracil; } \\
\text { iv }=\text { intravenous; } \mathrm{kg}= \\
\mathrm{NS}=\text { non-significant; } \\
\mathrm{VBL}=\text { vinblastine; } \mathrm{vs} . \\
\mathrm{a}=\text { Only statistically si } \\
\text { months. } \mathrm{e}=\text { Data extra } \\
\text { response rate at } 6 \mathrm{mo} \\
\mathrm{MU} / \mathrm{m}^{2} . \mathrm{h}=\text { On } 3 \text { cons } \\
6 \text { received recombinar } \\
\text { trial was not used in th } \\
\text { and one in each of the }\end{array}$ & 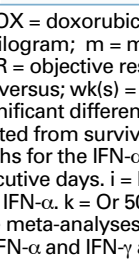 & $\begin{array}{l}\text { in; CIS = cisplatin; CR } \\
\text { letres; mg = milligram } \\
\text { sponse; po = per oral; } \\
\text { week(s); } \mathrm{yr}=\text { year. } \\
\text { ces are presented. } \mathrm{b}= \\
\text { al curve. } \mathrm{f}=\text { Overall res } \\
\text { and MPA was } 8 \% \text { anc } \\
\text { First } 10 \text { patients receiv } \\
\text { Omg } / \mathrm{m}^{2} \text { if creatinine cl} \\
\text { all three arms have b } \\
\text { arms, type of response }\end{array}$ & $\begin{array}{l}=\text { complete resp } \\
\text { 1s; MMC = mitor } \\
\text { PR = partial res } \\
\text { This trial had tw } \\
\text { sponse rate is ba } \\
\text { 1 } 1 \%, p=<0.001, r \\
\text { ed an initial dose } \\
\text { earance rate was } \\
\text { een included he } \\
\text { not specified. }\end{array}$ & $\begin{array}{l}\text { se; } d= \\
\text { cin C; } \\
\text { ise; } q= \\
\text { ther ar } \\
\text { d on } 15 \\
\text { pective } \\
\text { f } 50 \mathrm{MU} \\
\text { etween } \\
\text { as data }\end{array}$ & $\begin{array}{l}\text { IFN- } \alpha= \\
=\text { medrc } \\
\text { ery; RCTs } \\
\text { which ar } \\
\text { atients an } \\
=\text { = For pa } \\
2 \text { tiw, hov } \\
\text { and } 50 \mathrm{ml} \\
\text { esponse }\end{array}$ & $\begin{array}{l}\text { erferon- } \\
\text { proges } \\
\text { andom } \\
\\
\text { trepor } \\
\text { th pati } \\
\text { ts unak } \\
\text { er the } \\
\text { h or } 75 \\
\text { as not }\end{array}$ & $\begin{array}{l}\text {; IFN- } \gamma=i \\
\text { ne acetate } \\
\text { controlle } \\
\text { here as th } \\
\text { in the IFN } \\
\text { to tolerate } \\
\text { was lowe } \\
\mathrm{m}^{2} \text { if creat } \\
\text { ken down }\end{array}$ & $\begin{array}{l}\text { terferon-gar } \\
\text { mos }=- \text { mon } \\
\text { trials; } s c=s \\
y \text { are not in } \\
\alpha \text { and MPA } \\
\text { N- } \alpha \text { treatm } \\
\text { ed due exte } \\
\text { hine clearan } \\
y \text { trial arm. }\end{array}$ & $\begin{array}{l}\text { hma; im = } \\
\text { hs; } \mathrm{MU}= \\
\text { lbcutaneo } \\
\text { luded in th } \\
\text { reatment } \\
\text { nt at a do } \\
\text { sive side } \\
\text { e was gre } \\
\mathrm{n}=\text { Two re }\end{array}$ & $\begin{array}{l}\text { tramuscul } \\
\text { Million Unit } \\
\text { s; tiw = thr } \\
\text { e meta-ana } \\
\text { roups at } 12 \\
\text { of } 18 \mathrm{MU} \\
\text { ffects. } \mathrm{j}=\mathrm{T} \\
\text { ter than } 50 \\
\text { sponses oc }\end{array}$ & $\begin{array}{l}\text { arly; IU = Ir } \\
; \text { NR = not } \\
\text { times a v } \\
\text { ysis. c = At } \\
\text { weeks, res } \\
\mathrm{m}^{2} \text {, the dos } \\
\mathrm{n} \text { people } \\
\mathrm{nl} / \mathrm{min} . \mathrm{I}= \\
\text { urred in th }\end{array}$ & $\begin{array}{l}\text { ternational } \\
\text { reported; } \\
\text { leek; } \\
3 \text { months. } \\
\text { ectively. T } \\
\text { e was reduc } \\
\text { ceived puri } \\
\text { Vhile this ar } \\
\text { combinati }\end{array}$ & $\begin{array}{l}\text { Jnits; } \\
=\text { At } 6 \\
\text { e overall } \\
\text { ed to } 9 \\
\text { ied, } \\
\text { n of the } \\
\text { in arm }\end{array}$ \\
\hline
\end{tabular}




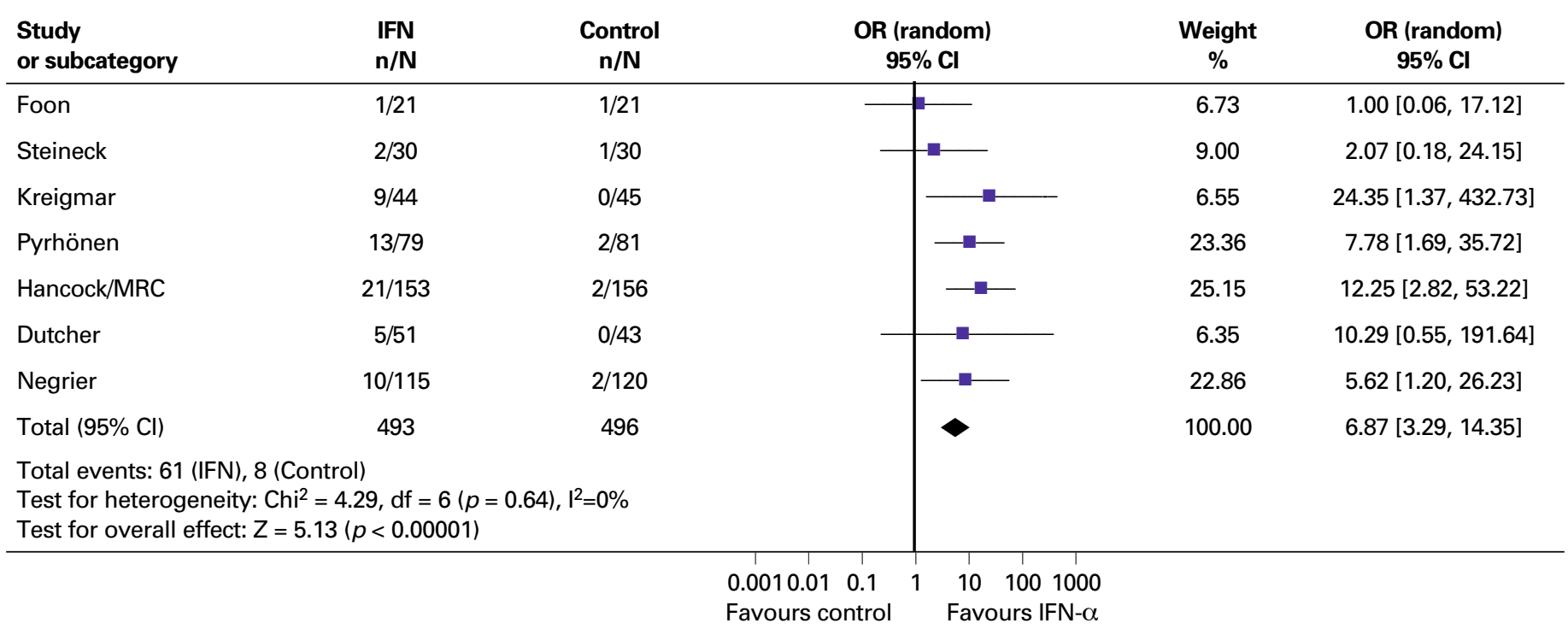

Fig. 1. Meta-analysis of response in randomized trials of interferon- $\alpha$ versus control in patients with inoperable renal cell carcinoma.

review. However, the new data provided often conflicted with the data presented in the original papers. Furthermore, 1 study reported 2 responses in the combination arm of the trial and one in each of the other arms but did not report the type of response. ${ }^{17}$ Two studies did not report mortality data. ${ }^{15,17}$ Study quality elements of the included trials are summarized in Table 1.

\section{Outcomes}

Outcome data from the RCTs for objective response rate, survival and disease progression are reported in Table 2 . All 3 RCTs reporting objective response rate showed a statistically significant difference in favour of IFN- $\alpha .{ }^{18-21}$

Two of the 5 trials providing survival data reported statistically significant differences in overall survival favouring IFN- $\alpha .{ }^{18,19,21}$ Hancock and colleagues reported longer median survival for patients receiving IFN- $\alpha$ than MPA (9 vs. 6.75 months, $p=0.013){ }^{18,19}$ Pyrhönen and colleagues reported that patients receiving IFN- $\alpha-2$ a combined with VBL had a longer median survival than that of patients receiving VBL alone (15.5 vs. 8.7 months, $p=0.0049){ }^{21}$ Of the 3 remaining trials, 1 found no difference between trial arms, ${ }^{10-12}$ and 2 did not provide statistical comparisons. ${ }^{15,16}$ A sixth trial reported no difference in median survival between groups, but did not provide data. ${ }^{20}$ One-year survival data were reported in 2 trials ${ }^{18,19,21}$ and extracted from survival curves in 4 trials. ${ }^{10-12,16,20,22}$ Two trials did not report survival outcomes. ${ }^{15,17}$

Disease progression was assessed in 3 trials. ${ }^{16,18,19,21}$ The trial by Pyrhönen and colleagues reported that IFN- $\alpha$ $2 a$ in combination with VBL demonstrated significantly longer progression-free survival than did treatment with VBL alone (3 vs. 2.1 months, $p=0.0001$ ). ${ }^{21}$
The adverse effects of IFN- $\alpha$ were reasonably consistent from trial to trial, although they increased in intensity and frequency with increased IFN- $\alpha$ dose. Reports of the toxicities from a large RCT as representative of IFN- $\alpha$ toxicity ${ }^{18,19}$ showed increased rates of lack of appetite (51\%), tiredness $(68 \%)$, nausea/vomiting (26\%/9\%), lack of energy (65\%), dry mouth $(41 \%)$, shivering $(23 \%)$ and depressed mood $(25 \%)$ with IFN- $\alpha$ after 4 weeks of treatment. Increased rates of lack of appetite, tiredness, lack of energy, dry mouth and shivering persisted at 12 weeks. Other reported symptoms included irritability, worrying, sore muscles, general pain, nervousness, despondent or tense feelings, difficulty sleeping, headaches, dizziness, decreased sexual interest, restlessness, anxiety, constipation, diarrhea, tingling in hands and feet, difficulty concentrating, sore mouth, loss of hair, shortness of breath, hoarseness and burning eyes.

\section{Meła-analysis}

Overall response and mortality were considered the primary endpoints for meta-analysis. Because of the previously mentioned issues with data consistency, study authors and the author of the Cochrane review were contacted to determine the data on which to base results. Responses from the authors were used in some cases as opposed to the data reported in trial reports for the meta-analysis.

The response data from 7 trials were pooled in a metaanalysis. ${ }^{10-12,16-22}$ The number of patients randomized to each treatment arm was not available for 1 trial; ${ }^{15}$ therefore, this trial was not included in the meta-analysis. The results of the meta-analysis appear in Fig. 1. The metaanalysis of the 7 trials produced an OR of $6.87(95 \% \mathrm{Cl}$, 3.29-14.35; $p<0.00001)$. 


\begin{tabular}{|c|c|c|c|c|}
\hline $\begin{array}{l}\text { Study } \\
\text { or subcategory }\end{array}$ & log [Hazard ratio] (SE) & $\begin{array}{l}\text { Hazard ratio (random) } \\
\qquad 95 \% \mathrm{Cl}\end{array}$ & $\begin{array}{l}\text { Weight } \\
\%\end{array}$ & $\begin{array}{l}\text { Hazard ratio (random) } \\
\qquad 95 \% \mathrm{Cl}\end{array}$ \\
\hline Steineck & $0.0488(0.2522)$ & 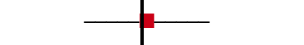 & 7.61 & $1.05[0.64,1.72]$ \\
\hline Kreigmar & $-0.4005(0.3044)$ & $\longrightarrow-$ & 5.24 & $0.67[0.37,1.22]$ \\
\hline Pyrhönen & $-0.4308(0.1685)$ & $\longrightarrow$ & 16.88 & $0.65[0.47,0.90]$ \\
\hline Hancock/MRC & $-0.3011(0.1090)$ & $\rightarrow-$ & 39.35 & $0.74[0.60,0.92]$ \\
\hline Dutcher & $-0.1278(0.2200)$ & $\longrightarrow$ & 9.98 & $0.88[0.57,1.35]$ \\
\hline Negrier & $-0.0459(0.1510)$ & $\rightarrow-$ & 20.93 & $0.96[0.71,1.28]$ \\
\hline \multicolumn{2}{|l|}{ Total $(95 \% \mathrm{Cl})$} & $\bullet$ & 100.00 & $0.79[0.69,0.91]$ \\
\hline \multicolumn{5}{|c|}{$\begin{array}{l}\text { Test for heterogeneity: } \mathrm{Chi}^{2}=5.08, \mathrm{df}=5(p=0.41), \mathrm{I}^{2}=1.6 \% \\
\text { Test for overall effect: } Z=3.30(p=0.0010)\end{array}$} \\
\hline
\end{tabular}

Fig. 2. Meta-analysis of mortality in randomized trials of interferon- $\alpha$ versus control in patients with inoperable renal cell carcinoma.

Mortality data suitable for meta-analysis were reported in 6 trials and combined in a meta-analysis. ${ }^{10,16,18-22}$ The results of the meta-analysis appear in Fig. 2. The HR for mortality after treatment with IFN- $\alpha$ was $0.79(95 \% \mathrm{Cl}, 0.69-$ $0.91 ; p=0.001)$.

\section{Discussion}

This review identified 8 RCTs that directly evaluated the use of IFN- $\alpha$ in locally advanced or metastatic RCC. These trials compared IFN- $\alpha$ alone or in combination with control therapies considered to have little or no activity in RCC. In our meta-analysis of 6 trials, the overall HR for death was 0.79 , indicating a $21 \%$ reduction in the risk of death for patients treated with IFN- $\alpha$ over the time periods of follow-up of the RCTs. Toxicity was higher with IFN- $\alpha$ therapy and, unfortunately, health-related quality of life was not evaluated. However, the odds of objective response were almost 7 times higher for patients receiving IFN- $\alpha$ containing regimens $(4.4 \%$ to $20 \%$ ) compared with patients in control groups (0 to $3 \%$ ). Heterogeneity was minimal in these analyses. None of the included RCTs used placebo control groups, which is an inherent limitation of this data set. This could potentially exaggerate survival differences between IFN- $\alpha$ and control due to the detrimental effects of control therapy, a development that was more likely to occur when chemotherapy was the control. Medroxyprogesterone acetate and IFN- $\gamma$ have not shown detrimental effects on survival, and analysis of these trials alone shows similar pooled survival results (data not shown). Only 1 trial included in the analyses used chemotherapy (single-agent $\mathrm{VBL}$ ) as control. ${ }^{21}$ This trial did have the most extreme HR for overall mortality, favouring IFN- $\alpha$ therapy. However, VBL has a low objective response rate in RCC $(7 \%),{ }^{23}$ is considered a mild cytotoxic drug, and was also included in combination with IFN- $\alpha$ in the experimental arm of this trial. A sensitivity analysis excluding the trial provided an overall HR for death of $0.83(95 \% \mathrm{Cl}, 0.71$ $0.96, p=0.01)$. The limitations of published data metaanalysis have been well-described and are potentially applicable to these results; ${ }^{24}$ nevertheless, we consider the results a comprehensive and robust synthesis of the best currently available clinical data.

Overall, toxicity appeared worse with IFN- $\alpha$ compared with non-IFN- $\alpha$ therapy. Toxicity for IFN- $\alpha$ is well-known and consistent from trial to trial; therefore, we presented toxicity data from a large randomized trial that reported increased rates of lack of appetite, tiredness, lack of energy, dry mouth and shivering with IFN- $\alpha$ after 12 weeks of treatment. ${ }^{18,19}$ No toxic deaths were reported; however, these data were not reported in most of the studies. The general opinion is that IFN- $\alpha$ regimens are associated with substantial toxicity; the magnitude of this toxicity may be underestimated in clinical trials due to patient selection factors, such as performance status and under-reporting.

Doses and modality of administration of IFN- $\alpha$ differed across the trials. In 5 trials, IFN- $\alpha$ was administered subcutaneously at doses ranging from 2 to $10 \mathrm{MU} / \mathrm{m}^{2}$ on a thriceweekly schedule. ${ }^{10-12,16-20}$ In 2 trials, IFN- $\alpha$ was administered intramuscularly at doses ranging from 3 to 10 $\mathrm{MU} / \mathrm{m}^{2}, 15,22$ and in another it was administered either subcutaneously or intramuscularly starting at $3 \mathrm{MU}$ and increasing to $18 \mathrm{MU} .{ }^{21}$ Whether there is a dose response to IFN- $\alpha$ is unclear; however, it is likely that toxicity is dependent on dose and schedule. In addition, there is no evidence of a difference in efficacy between recombinant IFN- $\alpha-2$ a and IFN- $\alpha-2 b$, or clear evidence of benefit of adding chemotherapy to IFN- $\alpha$. In view of this, the consensus of the authors was that it is reasonable to use the dose and schedule from the largest RCT showing benefit. ${ }^{18,19}$ This trial gave an ini- 
tial dose of $5 \mathrm{MU}$ subcutaneously followed by $10 \mathrm{MU}$ subcutaneously on a thrice-weekly schedule for a total of 12 weeks, unless progressive disease or objective response was seen. Treatment could be continued after 12 weeks in responding patients. In view of these data and the toxicities of IFN- $\alpha$, the value of treatment beyond 12 weeks in non-responding patients is questionable and should be considered on an individual basis.

Despite many years of research, the prognosis for patients with inoperable locally advanced or metastatic RCC had not changed until recently, and very few therapeutic options existed for these patients. Our synthesis of the data from randomized trials of IFN- $\alpha$-based immunotherapy confirms that IFN- $\alpha$ has anti-tumour activity in RCC, provides a genuine if modest survival benefit in this patient population, and should be considered as a potential treatment option. Evidence from randomized trials of angiogenesis inhibitors (i.e., sunitinib, sorafenib and temsirolimus) show that these agents are of superior clinical effectiveness to IFN- $\alpha$, with acceptable toxicity. The clinical benefits observed with these agents make them the preferred treatment modality. In particular, the low objective response rate (ORR) seen with IFN- $\alpha(6 \%$ to $20 \%)$ suggests that drugs, such as sunitinib (ORR $=33 \%$ to $40 \%$ ), may be preferred in patients with disease involving critical organs where prompt disease shrinkage to secure survival may be necessary.

As not all patients may have access to the newer angiogenesis therapies due to their costs, information about the effectiveness of IFN- $\alpha$ is still of value. Despite IFN- $\alpha$ and recent advances with other new drugs, patients with inoperable locally advanced or metastatic RCC continue to have an incurable malignancy, and further research to improve disease control and cure is necessary.

\section{Conclusion}

Until recently, very few systemic therapeutic options existed for patients with inoperable locally advanced or metastatic RCC. Immunotherapy with IFN- $\alpha$ can be considered a treatment option to modestly improve survival and disease control in this patient population. However, given the toxicity profile of IFN- $\alpha$, patient factors, such as age and performance status, must be taken into consideration and may affect patients' ability to tolerate therapy and benefit from it. Further, angiogenesis inhibitors have expanded the treatment repertoire for RCC and appear to have superior effectiveness compared to IFN- $\alpha$. In view of this, the role of IFN- $\alpha$ in the treatment of RCC is less clear. However, as not all patients may have access to the newer therapies due to the cost of these therapies, information about the effectiveness of IFN- $\alpha$ is still of value.

Locally advanced or metastatic RCC remains an incurable disease, current treatments remain palliative, and fur- ther research is warranted. Whenever possible, patients should be encouraged to participate in clinical trials.

*The Ottawa Hospital Regional Cancer Centre, Ottawa, ON; † Juravinski Cancer Centre, Hamilton, ON; : Cancer Care Ontario Program in Evidence-based Care, McMaster University, Hamilton, ON; $\S$ London Health Sciences Centre, London, ON

Competing interests: The members of the Genitourinary Cancer Disease Site Group disclosed potential conflicts of interest relating to this systematic review. One author (SH) reported grant/research support from two companies with competing treatments, and serving on an advisory board for one company with a competing treatment. One author $(\mathrm{CC})$ reported serving on an advisory board for two companies with competing treatments. No further conflicts were declared by the authors.

Acknowledgements: The Genitourinary Cancer Disease Site Group would like to thank Dr. Christina Canil, Dr. Sebastien Hotte and Dr. Eric Winquist for taking the lead in drafting this systematic review with meta-analyses. The authors would also like to thank Dr. Janice Dutcher, Ms. Judi Manola, and Ms. Sylvie Negrier for their personal correspondence clarifying data, Dr. Chris Coppin for his correspondence concerning his review, Ms. Kate Bak for contributions to an early version of this report, and Ms. Cindy Walker-Dilks for assistance in preparing this manuscript.

This paper has been peer-reviewed.

\section{References}

1. Canadian Cancer Society, National Cancer Institute of Canada. Canadian Cancer Statistics 2009. Toronto (Canada): National Cancer Institute of Canada; 2009.

2. Bukowski RM. Immunotherapy in renal cell carcinoma. Oncology 1999;13:801-10.

3. Rabinovitch RA, Zelefsky MJ, Gaynor JJ, et al. Patterns of failure following surgical resection of renal cell carcinoma: implications for adjuvant local and systemic therapy. J Clin Oncol 1994;12:206-12.

4. Amato R. Chemotherapy for renal cell carcinoma. Semin Oncol 2000;27:177-86.

5. Yagoda A, Abi-Rached B, Petrylak D. Chemotherapy for advanced renal cell carcinoma: 1983-1993. Semin Oncol 1995;22:42-60.

6. Oliver RT, Nethersell AB, Bottomley JM. Unexplained spontaneous regression and alpha-interferon as treatment for metastatic renal cell carcinoma. Br J Urol 1989;63:128-31.

7. Bukowski RM. Cytokine therapy for metastatic renal cell carcinoma. Semin Urol Oncol 2001;19:148-54.

8. Fosså SD. Interferon in metastatic renal cell carcinoma. Semin Oncol. 2000;27:187-93.

9. Coppin C, Porzsolt F, Awa A, et al. Immunotherapy for advanced renal cell cancer. Cochrane Database Syst Rev 2005; Issue 2. D01:10.1002/14651858.CD001425.pub2.

10. Negrier S. Cytokines in metastatic renal cell carcinoma: conclusions from the French PERCY program [abstract]. Ann Oncol 2006;17:ix32.

11. Negrier S, Perol D, Ravaud A, et al. Do cytokines improve survival in patients with metastatic renal cell carcinoma (MRCC) of the intermediate prognosis? Results of the prospective randomized PERCY Quattro trial. Proc Soc Clin Oncol 2005;23:380s.

12. Negrier $S$, Perol D, Ravaud A, et al.; for the French Immunotherapy Intergroup. Medroxyprogesterone, interferon alfa-2a, interleukin 2, or combination of both cytokines in patients with metastatic renal carcinoma of intermediate prognosis: results of a randomized controlled trial. Cancer 2007;110:2468-77.

13. Parmar $M$, Torri V, Stewart L. Extracting summary statistics to perform meta-analysis of the published literature for survival endpoints. Stat Med 1998; 17:2815-34.

14. Hernberg M, Pyrhönen S, Muhonen T. Regimens with or without interferon-a as treatment for metastatic melanoma and renal cell carcinoma: an overview of randomized trials. J Immunother 1999;22:145-54.

15. Dexeus F, Logothetis C, Sella A, et al. Interferon alternating with chemotherapy for patients with metastatic renal cell carcinoma. J Clin Oncol 1989; 12:350-4.

16. Dutcher JP, Fine JP, Krigel RL, et al. Stratification by risk factors predicts survival on the active treatment arm in a randomized phase II study of interferon-gamma plus/minus interferon-alpha in advanced renal cell carcinoma (E6890). Med Oncol 2003;20:271-81. 
Canil et al.

17. Foon $\mathrm{K}$, Doroshow J, Bonnem E, et al. A prospective randomized trial of alpha 2B-interferon/gamma-interferon or the combination in advanced metastatic renal cell carcinoma. J Biol Response Mod 1988;7:540-5.

18. Hancock B, Griffiths $G$, Ritchie $A$, et al. Updated results of the MRC randomised controlled trial of alpho interferon vs. MPA in patients with metastatic renal carcinoma [abstract]. Proc Am Soc Clin Oncol 2000;19:340a.

19. Medical Research Council Renal Cancer Collaborators. Interferon-a and survival in metastatic renal carcinoma: early results of a randomized controlled trial. Lancet 1999;353:14-7.

20. Kriegmair $M$, Oberneder $R$, Hofstetter $A$. Interferon alfa and vinblastine versus medroxyprogesterone acetate in the treatment of metastatic renal cell carcinoma. Urology 1995;45:758-62.

21. Pyrhönen $S$, Salminen E, Lehtonen $T$, et al. Prospective randomized trial of interferon alfa-2a plus vinblastine versus vinblastine alone in patients with advanced renal cell cancer. J Clin Oncol 1999;17:2859-67.
22. Steineck G, Strander $H$, Carbin B, et al. Recombinant leukocyte interferon alpha-2a and medroxyprogesterone in advanced renal cell carcinoma. A randomized trial. Acta Oncol 1990;29:155-62.

23. Hartmann J, Bokemeyer C. Chemotherapy for renal cell carcinoma. Anticancer Res 1999;19:1541-3.

24. Egger $M$, Dickerson K, Smith GD. Problems and limitations in conducting systematic reviews. In: Egger M, Smith GD, Altman D, eds. Systematic Reviews in Health Care: Meta-analysis in Context. 2nd ed. London: BMJ Publishing Group; 2001:43-68.

Correspondence: Dr. Christina Canil, The Ottawa Hospital Regional Cancer Centre, 503 Smyth Rd., Ottawa, ON K1H 1C4; fax: 613-247-3511; ccanil@ottawahospital.on.ca 